\title{
Intermittency of storms and substorms: is it related to the critical behaviour?
}

\author{
P. Dobias ${ }^{1}$ and J. A. Wanliss ${ }^{2}$ \\ ${ }^{1}$ DRDC CORA, 101 Colonel By Dr., Ottawa, ON, K1A0K2, Canada \\ ${ }^{2}$ Presbyterian College, 503 S Broad St, Clinton, SC 29325-2865, USA
}

Received: 1 September 2008 - Revised: 14 April 2009 - Accepted: 14 April 2009 - Published: 4 May 2009

\begin{abstract}
Intermittency is one of the possible means of quantifying dynamics of fractal processes. In this paper, the analysis of the intermittency of magnetospheric storms and substorms is presented. The analysis allows for a classification of the processes in terms of the power-law scaling of the magnitude of deviations of the index values from the values at quiet times (normal state), and the relative timings of occurrences of such deviations. These are expressed in terms of the co-dimension and the Fano factor. The relationship between the two is related to the nature of the processes behind the observed storm and substorm dynamics. The results suggest that there is a similarity between the two, and therefore it is possible that there are common dynamical processes behind the storms and substorms. In particular, it appears that both of them behave consistently with what would be expected for critical systems, which is consistent with the conclusions of several previous works.
\end{abstract}

Keywords. Electromagnetics (Plasmas) - Interplanetary physics (Instruments and techniques; General or miscellaneous)

\section{Introduction}

There have been ongoing arguments about the relationship between the nature of magnetospheric storms and substorms, and about the triggering mechanisms for both of them. Wanliss and Dobias (2007) suggested that the dynamical properties of the DST index, in particular the variations between quiet and storm times, are consistent with nonequilibrium phase transition-like dynamics. Similar analyses were performed for substorms (Ukhorskiy et al., 1997; Sitnov et al.,

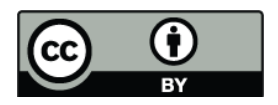

Correspondence to: P. Dobias

(peter.dobias@drdc-rddc.gc.ca)
2001; Uritsky and Pudovkin, 1998; Lui et al., 2001). If substorms did indeed feature critical behavior, this might explain ongoing disagreements as to the substorm trigger, with some observations pointing to a near-earth trigger such as field-line resonances (Samson et al., 2003) and other observations pointing to the near-earth neutral line (NENL) and braking of earthward flows (Shiokawa et al., 1997) as the cause. Similarly, it would explain sometimes contradictory evidence as to the relationship between the solar wind dynamics and storms (Wanliss and Weygand, 2007).

In this paper, the properties of the storm $(\mathrm{Sym}-\mathrm{H})$ and substorm (AE and AL) indices (see Sect. 2) are explored with regard to intermittency to further explore the possibility of the presence of critical behaviour. The Sym-H index is a representative of the low-latitude and the $\mathrm{AE}$ and $\mathrm{AL}$ indices of the high-latitude geomagnetic disturbances. To establish whether it is likely that storms and substorms are fractal processes the three indices are tested for a presence of the powerlaw relationship between the size and the frequency of occurrences of such events.

The majority of works related to intermittency of the magnetospheric dynamics deals with the intermittency in terms of the turbulent process. For example, Angelopoulos et al. (1999) found that both the bursty bulk flow events and the ambient flows could be described as an intermittent turbulence. Lui et al. (2001) found magnetic turbulence in the geomagnetic tail with the intermittency that varies with the distance from the neutral sheet. Stepanova et al., (2005a, 2005b) have found that the intermittency has strong magnetic local time behaviour being significantly stronger close to the substorm eye. Weygand et al. (2005) have also found that both the scaling behavior of the probability distribution functions and the multifractal structure function suggest that intermittent MHD turbulence is present within the plasma sheet. This paper strives to look at the intermittency of the magnetospheric dynamics from a slightly different angle. It investigates the intermittency of the temporal behaviour of selected

Published by Copernicus Publications on behalf of the European Geosciences Union. 


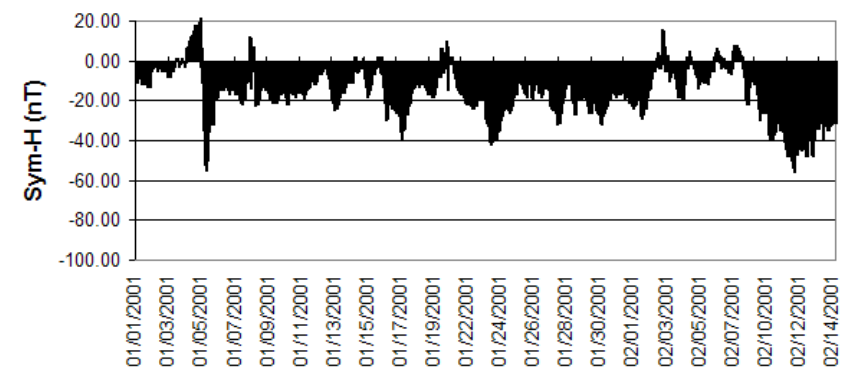

Fig. 1. SYM-H index for the period between 1 January and 15 February 2001 (collected at $1 \mathrm{~min}$ intervals). Only negative values are shown.

magnetospheric indices in order to establish a possibility of the presence of critical behaviour in the magnetosphere.

The paper is organized as follows. At first, the data sources are discussed, then a brief summary of intermittency and fractal point processes, as well as the classification of point processes in terms of intermittency are presented. Following this, the frequency distribution of the indices is analyzed to establish the related fractal nature of storms and substorms. Afterwards, the intermittency of the Sym-H, AE, and AL indices is analyzed, and the results are interpreted in terms of point processes. Finally, the fractal nature of storms and substorms is compared, and the implications of the findings are discussed.

\section{Data}

The data used in this study comprise sources from low and high-latitudes. For low-latitudes the SYM-H index, one of the important space storm indices, is utilized. This is the 1min high-resolution version of DST (Wanliss and Showalter, 2006). This index is considered to reflect variations in the intensity of the symmetric part of the ring current that circles Earth at altitudes ranging from about 3 to 8 Earth radii $\left(R_{E}\right)$, and is proportional to the total energy in the drifting particles that form the ring current (Dessler and Parker, 1959; Sckopke, 1966). The index is formed from magnetic observatories close enough to the magnetic equator that they are not strongly influenced by auroral current systems. Second, they are far enough away from the magnetic equator so that they are not significantly influenced by the equatorial electrojet current that flows in the ionosphere. They are also relatively evenly spaced in longitude. The convolution of their magnetic variations forms the SYM-H index, measured in $\mathrm{nT}$, which is considered to provide a reasonable global estimate of the variation of the horizontal field near the equator.

In the sense that they are constructed from multiple magnetometer stations spanning 360 degrees in longitude, SYM$\mathrm{H}$ indices can be considered global geomagnetic activity indicators. However, they represent rather localized effects in that all the component stations are in or near the auroral oval. The ionospheric current systems whose magnetic perturbations they measure are almost exclusively at high latitudes, so SYM-H indices provide limited information about the behavior at low- and mid-latitudes, regions that map to the ring current, near-earth plasma sheet and other neighborhoods in the magnetosphere with relatively dense plasma. The SYM$\mathrm{H}$ index is known to have two main components, one directly driven by the solar wind and the other related to the magnetotail unloading process (Bargatze et al., 1985). The cadence of SYM-H is 1 min. The other two indices used are AL and AE that characterize high latitude dynamics relevant for substorms. There are actually four auroral electrojet indices: Auroral Lower (AL) Auroral Upper (AU), the other two are the linear combination of previous ones: $\mathrm{AE}=(\mathrm{AU}-\mathrm{AL}) / 2$; $\mathrm{AO}=(\mathrm{AU}+\mathrm{AL}) / 2$. In this paper we use the $\mathrm{AE}$ and $\mathrm{AL}$ indices. The AL index in particular is the most suitable index for studying the dynamics of magnetospheric substorms. With these two different types of data, one clearly related to storms and the other to substorms, it is possible to gain some understanding of the behaviour of certain magnetospheric variations, and to capture a measure of the magnetospheric fluctuations from the low- to high-latitudes to which they map along magnetic field lines. The data used in the analysis covered the period from 1 January to 15 February 2001. During this period the magnetosphere was in a slightly perturbed state with few periods of storms activity (Fig. 1), and a number of substorms (Figs. 2, 3). Furthermore, there appears to be a significant degree of correlations between the two indices for the analyzed period.

\section{Stochastic point processes and intermittency}

Natural processes with a long-term memory require a mathematical description with fractal (power-law) statistics. This includes processes in physics, biology, and medical sciences (Bickel, 1999). Two classes of such discrete processes that are of potential interest here are the fractal point process (FPP) (Lowen and Teich, 1995) and the fractal rate point process (FRPP) (Thurner et al., 1997).

In general, a point process is defined as a representation of a sequence of events on a time line. The methodology used below applies to stationary point processes, which are defined as point processes independent of the time origin $t_{0}$. In terms of distributions $N(t, t+T)$ :

$N\left(t_{0}, t_{0}+T\right)=N(0, T) \equiv N(T)$

One of the important characteristics of point processes is intermittency. A sequence of events is called intermittent if its dynamics deviate at times from the usual behaviour (Bickel, 1999). Sudden, positive deviations increase the mean of a sequence to values much higher than the most probable value. An example of such dynamics can include sudden spikes in a child's activity as in Bickel (1999), avalanches in sand-piles, 


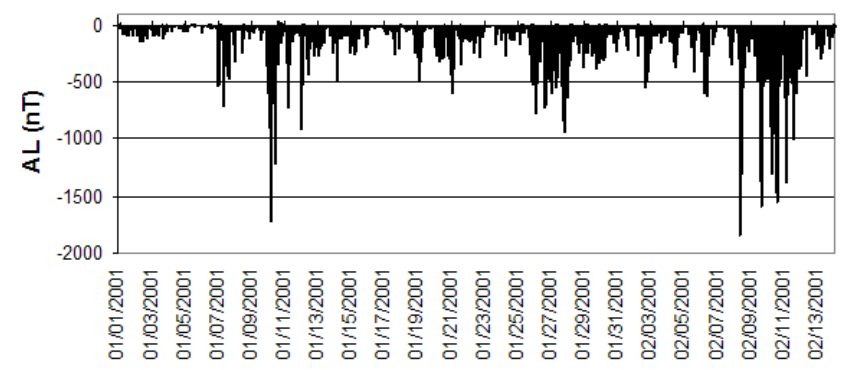

Fig. 2. AL index for the period between 1 January and 15 February 2001 (collected at $1 \mathrm{~min}$ intervals).

or possibly sharp increases (decreases) in $\mathrm{AE}$ and $\mathrm{AL}$ indices during substorms and SYM-H index during storms as is discussed in this paper.

The definition of the intermittency used in this paper (Bickel, 1999) is applicable to critical systems, and is different from the definition typically used by the turbulence community (see e.g. Stepanova et al., 2003). However, this not represent a major limitation, since this paper does not address the relationship between the turbulence and criticality. Bickel (1999) proposed the use of the correlation codimension as a measure of intermittency. The method of calculation applicable to the casualty numbers is briefly summarized here. The correlation co-dimension $C_{2}$ is related to the second moment of the counting process $N$ via a power law:

$\left\langle N^{2}(T)\right\rangle \sim T^{2-C_{2}}$

The correlation co-dimension values range between 0 and 1 . The value of 0 corresponds to a non-intermittent (continuous) process, while the value of 1 corresponds to maximum intermittency (a solitary event). Typical intermittent processes have values between the two extremes which implies that they are fractal processes. The co-dimension is calculated as follows.

It is assumed that there is an experimental time series $Z_{1}, Z_{2}, \ldots, Z_{n}$ sampled at time steps $T_{0}$. At first, a counting process is created:

$N_{k}=\sum_{i=1}^{k} Z_{i}, \quad k=1,2, \ldots, n$

Afterwards, the variance $\left\langle N^{2}(T)\right\rangle$ at times $T=k T_{0}$ is estimated using maximum-overlap estimator $S_{k}$ for all $k=1,2, \ldots, k_{\max } \leq n:$

$S_{k}=\left\langle N_{k}^{2}\left(k T_{0}\right)\right\rangle=\frac{1}{n-k+1} \sum_{j=1}^{n-k+1}\left(N_{j+k}-N_{j}\right)^{2}$

Finally, the value of $2-C_{2}$ is estimated from the regression of the slope of $\log S_{k}$ vs. $\log k T_{0}$. For FPPs the co-variance

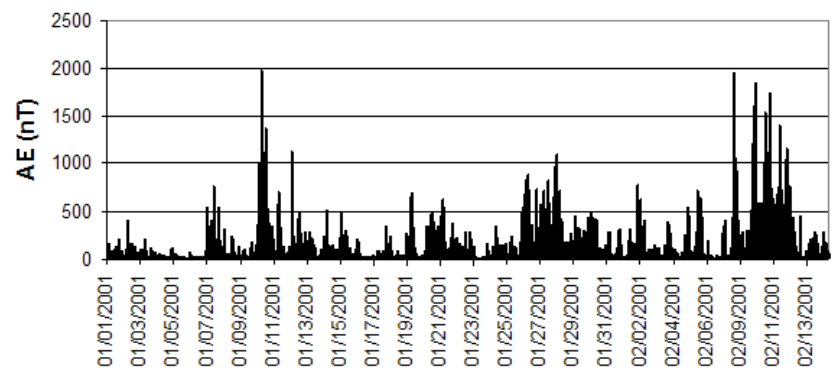

Fig. 3. AE index for the period between 1 January and 15 February 2001 (collected at 1 min intervals).

can be related to the Fano factor, defined as the ratio of the variance to the mean:

$F(T) \equiv \frac{\left\langle N^{2}(T)\right\rangle-\langle N(T)\rangle^{2}}{\langle N(T)\rangle}=T^{\alpha}$

Comparison with Eq. (2) yields equation connecting codimension with Fano factor:

$\alpha=1-C_{2}$

On the other hand, for FRPPs Eq. (6) does not hold for FRPP. If there are long-term correlations present in the time series (such as for fractional Brownian motion, or for critical systems), the Fano factor is related to the Hurst coefficient $H$ (Hurst, 1951) as (Bickel, 1999):

$H=\frac{1+\alpha}{2}$

Equation (7) only makes sense if $H>0.5$, hence the requirement for the correlated system.

The Fano factor can be estimated in a fashion similar to the calculation for the co-dimension. For an experimental time series $Z_{1}, Z_{2}, \ldots, Z_{n}$ the counting process (3) is used to estimate the Fano factor using maximum overlapping windows (Bickel, 1999):

$$
\begin{aligned}
F_{k}= & \frac{1}{1 /(n-k+1) \sum_{j=1}^{n-k+1}\left(N_{j+k}-N_{j}\right)} \times \\
\times & \left\{\frac{1}{n-k} \sum_{j=1}^{n-k+1}\left(N_{j+k}-N_{j}\right)^{2}-\frac{1}{(n-k)(n-k+1)}\right. \\
& {\left.\left[\sum_{j=1}^{n-k+1}\left(N_{j+k}-N_{j}\right)\right]^{2}\right\} }
\end{aligned}
$$

At this point $\alpha$ is estimated from the regression of the slope of $\log -\log$ plot of $\log F_{k}$ vs. $\log k T_{0}$. As mentioned above, the Fano factor can be used to either verify if a process is an FPP (Eq. 6 holds), or to estimate the Hurst coefficient $H$ for correlated processes (typically with low intermittency). 

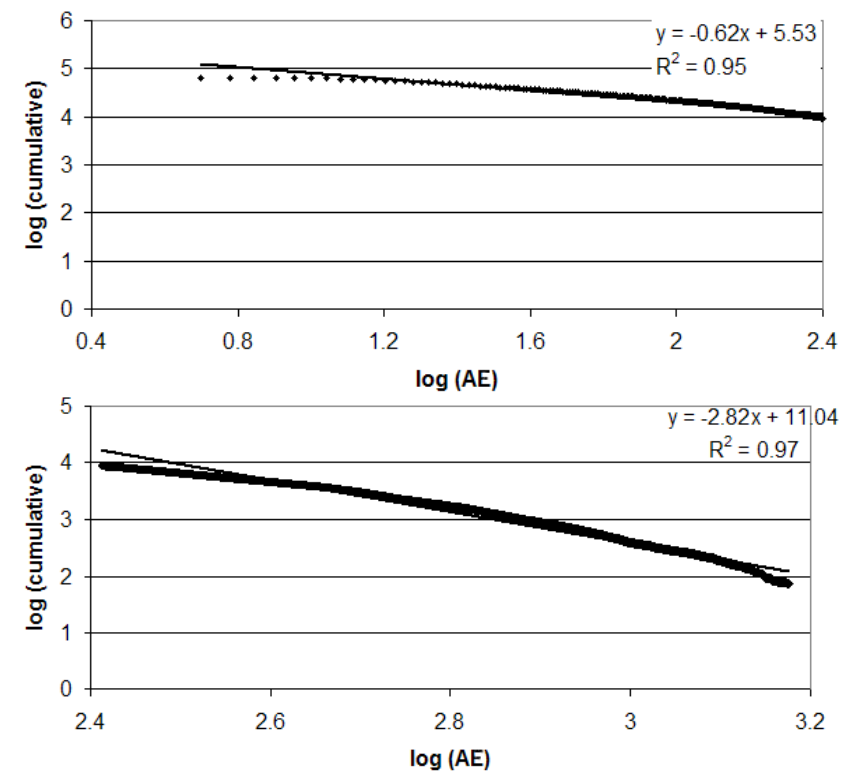

Fig. 4. Magnitude-frequency distribution for the AL index over $-800 \mathrm{nT}$ (quiet - top) and less than $-800 \mathrm{nT}$ (bottom).

Thus, the relationship between $C_{2}$ and $\alpha$ can be used to determine the class of point process, and for correlated systems $H$ can be estimated.

\section{Intermittency and criticality}

The idea of a connection between criticality, especially in the context of self-organized criticality, and intermittency is not new, and has been discussed in a number of papers (Contoyiannis and Diakonos, 2000; Halley et al., 2004; Murin et al., 1997). The fractal stochastic processes are typically characterized by a power-law size frequency distribution, related to the presence of long term memory in the system. However, power-law size frequency distributions are also typical for critical systems (Bak, 1988; Turcotte, 1997) in which case they are related to the breakdown of scale separation (the dynamical changes reach across spatial and temporal scales). Thus the intermittent behaviour seems to imply the approach of the system to criticality (Contoyiannis and Diakonos, 2000).

Furthermore, the power-law distribution implies the presence of long-term memory in the system. This is likely to lead to either persistence or anti-persistence in the system, and in turn to the presence of non-integer intermittency $\left(0<C_{2}<1\right)$. Transition from a random to a correlated state was actually observed during the active periods in the $D_{s t}$ index (Wanliss and Dobias, 2007). A system in which antipersistent behaviors dominates is likely to feature a higher intermittency, since the events are much sparser, and the normal behaviour is no activity. On the other hand, the persis- tence implies long-term correlations in the system behaviour, and thus lower intermittency.

Persistent point processes obey Eq. (7), which is consistent with the values of the Hurst coefficient $H>0.5$. Also, they typically feature lower intermittency. Higher intermittency of anti-persistent point processes is consistent with the sub-critical discharge-event systems (DES) (Woodard et al., 2006). The DES are not at the point of criticality yet, but they are in a near-critical state. In such cases energy build-up within the system is likely to lead to a sudden release that, unlike critical systems, does not lead to a complete reorganization of the system. In the context of high-latitudes, these kind of behaviors would refer to pseudo-breakups. These are high-latitude energy reorganizations that nevertheless do not progress to become full magnetospheric substorms. For antipersistent systems $(H<0.5)$ it is impossible to satisfy Eq. (7).

\section{Size-frequency distribution of AE, AL, and SYM-H indices}

As mentioned in Sect. 3, fractal processes are characterized by fractal (inverse power-law) size-frequency distributions. This means that the number $N(M)$ of events with magnitude of at least $M$ scales as

$N(M)=\Lambda M^{-b}$

Equation (9) can be alternatively expressed as a log-log relationship

$\log N(M)=a-b \log M$

To assess a possibility of the fractal nature of storm and substorm indices, the log-log plots of frequency versus value were plotted for the AE, AL and SYM-H indices. The results are shown in Figs. 4-6. All three indices feature what appears to be a bi-fractal distribution, with different powerlaw dependences for quiet and perturbed times. The slope for the quiet times is relatively shallow, suggesting that all of the possible values have comparable probabilities. On the other hand, the slope for the perturbed times is generally rather steep. The transition is fairly sharp for the SYM-H index, the variation in the slope of the power law appears to be at approximately $-30 \mathrm{nT}$. For the AE and AL index the transition is more distributed, ranging from the magnitude of several hundreds nT to about $1000 \mathrm{nT}$. It may be caused by the fact that the substorms are localized events, and therefore a global index such as AE or AL does not provide the clear distinction of the quiet and perturbed times. Since storms are global events, they have stronger and clearer signature in a global index such as the SYM-H. The change in the distribution suggests that there is a phenomenon akin to a nonequilibrium phase transition occurring in the system as it undergoes storm (or substorm) onset, changing the nature of the system (Wanliss and Dobias, 2007). These findings suggest a crossscale dynamics consistent with critical behaviour (Turcotte, 1997) for both storms and substorms. 

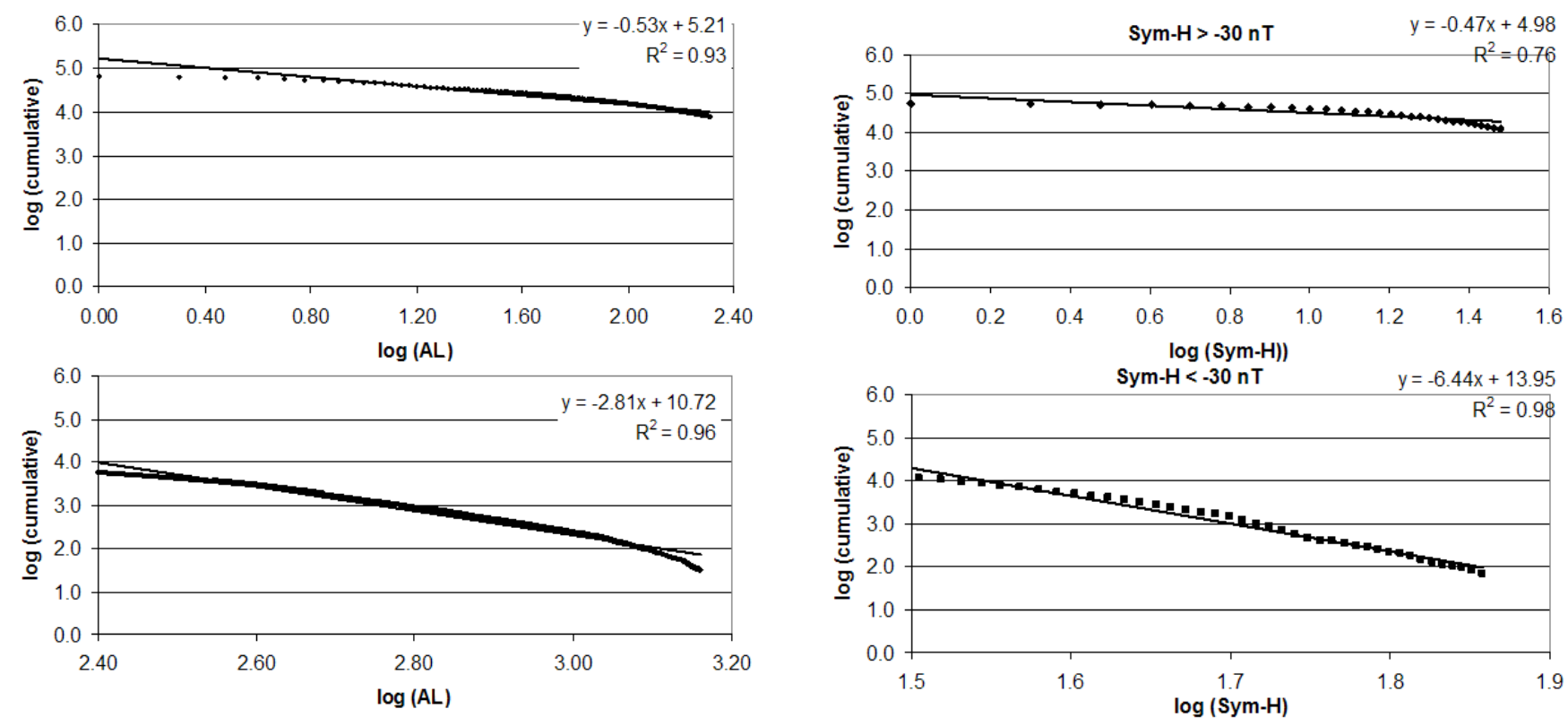

Fig. 5. Magnitude-frequency distribution for the AE index under $800 \mathrm{nT}$ (quiet - top) and over $800 \mathrm{nT}$ (bottom).

\section{Intermittency of storms and substorms}

As discussed in Sect. 4, there is possibly a relationship between intermittency and criticality. In this paper the intermittency in storm and substorm activity expressed in terms of SYM-H, AL, and AE indices is analyzed. The results are qualitatively similar for both storms and substorms.

\subsection{Storms}

On the basis of variations in the nature of long-term correlations between magnetospheric storms and quiet times, Wanliss and Dobias (2007) suggested that at the moment of a storm intensification stage the magnetosphere undergoes a kind of non-equilibrium phase transition. This finding is consistent with the observations made for the SYM-H index as outlined in the previous section. To further corroborate this possibility, the intermittency was estimated at first for the entire period of 1 January-15 February 2001, and then it was estimated separately for the times with SYM-H $<-30 \mathrm{nT}$ and $\mathrm{SYM}-\mathrm{H}>-30 \mathrm{nT}$ (quiet times). For the analyzed period there were about half a dozen of short storms, and one prolonged storm.

For the entire period, and for the quiet time the value for the co-dimension was found to be extremely low, with $C_{2}=0.03$. This suggests that the magnetospheric dynamics during this period was very uniform. The Fano factor for the entire analyzed period, and for the quiet times was $\alpha=0.81$. For the perturbed period, the co-dimension was $C_{2}=0.09$. While this implies relatively low intermittency, this was likely caused by the relatively limited storm activ-

Fig. 6. Magnitude-frequency distribution for the Sym-H index over $-30 \mathrm{nT}$ (quiet - top) and less than $-30 \mathrm{nT}$ (bottom).

ity, mainly focused in one long storm. The Fano factor was slightly higher than for the quiet times, $\alpha=0.89$, suggesting a high degree of correlations in the activity. Furthermore, while for the quiet periods the relationship (6) between the co-dimension and the Fano factor did not hold, for the storms it was satisfied, suggesting that the storms might fall to the category of FPPs.

While the change in the intermittency between quiet and stormy periods is not too dramatic, the fact that there is a difference in the relationship between the co-dimension and the Fano factor suggests that the magnetosphere is in a different statistical state during quiet times than during storms, which is consistent with the findings of Wanliss and Dobias (2007).

To account for the fact that storms happen at the timescale of days (rather than hours, as is the case with substorms), an additional analysis was performed using hourly data. In this case the index values were more intermittent, with $C_{2}=0.17$ for the entire timeseries. The Fano factor was $\alpha=0.55$. The low value of the Fano factor suggests that the quiet time values are fairly random. The storms were more intermittent, with the values of $C_{2}=0.25$, and the Fano factor $\alpha=0.74$. Again, this is consistent with Eq. (6) for an FPP. Thus there is a qualitative agreement between the 1-min and 60-min data.

\subsection{Substorms}

As is mentioned in Sect. 5, the frequency-size distribution of both the AE and AL indices exhibits the power-law dependence. There were only few spikes in these indices, and consequently the intermittency for the entire period (and the 
quiet time) was fairly low, but still higher than for the storms (observed in 1-min data). The co-dimension for the AE index for the entire analyzed period was $C_{2}=0.11$. The subsequent calculation revealed that the Fano factor $\alpha=0.71$ deviates during quiet times from the prediction of Eq. (6). The $\mathrm{AL}$ index features slightly higher intermittency than the $\mathrm{AE}$ index; the co-dimension for the overall period is $C_{2}=0.13$. The value for the Fano factor $\alpha=0.83$ is closer to the prediction of Eq. (6), but still varies from it.

The picture looks somewhat different for the substorm periods. The limit value for the analysis was taken from the break point of the power-law distribution, and was set to be $800 \mathrm{nT}$ for the AE, and $-800 \mathrm{nT}$ for the AL index. The substorm activity was fairly intermittent, with the values for the co-dimension being 0.56 for the $\mathrm{AL}$ and 0.68 for the $\mathrm{AL}$ index. The Fano factor was 0.47 for the AE, and 0.31 for the AE index. In both cases the Fano factor values are very close to the values predicted by Eq. (6). Thus, like storms, the substorms seem to behave as the FPPs, while the quiet times deviate from the characteristics of the FPPs.

\section{Storms and substorms as point processes}

The results for the intermittency of storms and substorms in Sect. 6, especially the consistency of the co-dimension $C_{2}$ and Fano factor $\alpha$ with Eq. (6), suggest that both storms and substorms behave consistently with the expectations for the FPPs. In 1-min data the intermittency of the storms for the observed period was fairly low, likely related to the fact that the storm activity takes place over days rather than hours. This claim is further supported by much higher values for the intermittency in 60-min data. In 1-min data, the substorm activity was much more intermittent when than the storm activity. This difference suggests that there is likely no direct relationship between the storm and substorm activity for the observed period. On the other hand, the commonality of the transition to an FPP for both storms and substorms suggests that there might be a similar underlying (phase transitionlike) dynamics for both types of processes.

For all three indices the values of the Fano factor, and in particular the increase in the values of the Fano factor for the perturbed periods suggest that there are strong temporal correlations in the magnetospheric system in a wide range of latitudes. However, it is impossible to use Eq. (7) to estimate the value of the Hurst coefficient, since both the storms and substorms behave as a FPP rather than a FRPP. For the storms, much more detailed analysis of the long term correlations has been done by Wanliss et al. (2005) and Wanliss and Dobias (2007). The latter work (Wanliss and Dobias, 2007) suggests that at least for the storms the perturbed periods are indeed characterized by a high degree of correlations. Conversely, the quiet periods are characterized by almost no correlations (they seem to be driven by the white noise). The correlations of the indices for the storms and substorms have a potentially important implication, suggesting a critical system in both instances.

\section{Conclusions}

The intermittency of the magnetospheric activity was analyzed using the SYM-H (relating to low latitudes and storms), AL and AE (relating to higher latitudes and substorms) indices. It was found that, in terms of their statistical properties, both storms and substorms have some common properties. While the substorm activity for the analyzed period was much more intermittent, the values of the Fano factor for both storms and substorms were consistent with the relation between the Fano factor and the co-dimension valid for the FPP. The results suggest a correlated behaviour during both storms and substorms. This factor, combined with the scaling properties between the size of events and their frequency, suggests that both storms and substorms function in a way consistent with the behavior of critical systems.

These findings are consistent with earlier works. Criticality is likely related to the availability of the free energy in the magnetosphere (Woodard et al., 2006). Once there is sufficient free energy accumulated in the plasma sheet, any perturbation (internal or external) can trigger a reorganization such as a storm or substorm (Dobias et al., 2007). This observation is consistent with seemingly contradictory observations of what causes substorms (FLRs, solar wind reversals, BBF) as well. These observations also speak to the fact that the community is still arguing about whether substorms are an in-out or an out-in process. Our results suggest that if one seeks a trigger then one will tend to find many different possible sources of a substorm onset; it is not the trigger that is so important as the energetic state of the high-latitude magnetosphere. Dobias et al. $(2004,2007)$ demonstrated that during the growth phase there is a significant reconfiguration of the free energy in the near-Earth plasma sheet. Using an assumption of FLR-like perturbations present in the system just prior to the onset, they demonstrated that the plasma sheet becomes explosively unstable at the time of the onset. However, the presence of FLRs was not a necessary condition for the instability, and any other similar perturbation would lead to the destabilization of the plasma sheet as well. This conclusion is consistent with the assumption that during the growth phase the magnetosphere is driven to a critical state, and at the moment of onset the reconfiguration happens. This is further consistent with the conclusions made in this paper that the substorms indeed seem to feature critical behaviour.

In earlier work it was proposed that space storms are a kind of nonequilibrium phase transition (Wanliss and Dobias, 2007). For example, rapid changes in the fractal correlations were observed near the initial phase of space storms. Such rapid changes in fractal scaling behavior are consistent with absorbing state phase transitions. This type of dynamics can be in turn possibly explained by the concept 
of self-organized criticality (Dickman et al., 1998; Griffiths, 1972). It is now becoming clearer that in terms of their statistical behavior storms and substorms are similar in many ways, though macroparameters and drivers appear so dissimilar.

This present study suggests further that there is a strong similarity between the critical nature of storms and substorms. This leads to a question: is there a common underlying dynamics? Many examples exist to distinguish the physics of storms and substorms, yet the observations made in this paper suggest that they may be in a similar class of physical processes. If the magnetosphere is in a critical or near-critical state, and has consistently similar statistical behaviors at low- and high-latitudes, then the search for universal parameters, such as energy or entropy, may be a step in the right direction. Future research could also address correlations between scaling of solar wind characteristics, as well as analyze longer time periods.

Acknowledgements. The authors would like to thank both reviewers for insightful and valuable comments.

Topical Editor R. Nakamura thanks M. Stepanova and another anonymous referee for their help in evaluating this paper.

\section{References}

Angelopoulos, V., Mukai, T., and Kokubun, S.: Evidence for intermittency in Earth's plasma sheet and implications for selforganized criticality, Phys. Plasmas, 6, 4161-4168, 1999.

Bak, P., Tang, C., and Wiesenfeld, K.: Self-organized criticality, Phys. Rev. A, 38, 364-374, 1988.

Bargatze, L. F., Baker, D. N., McPherron, R. L., and Hones, E. W.: Magnetospheric response to the IMF: substorms, J. Geophys. Res., 90, 6387-6394, 1985.

Bickel, D. R.: Estimating the Intermittency of Point Processes with Applications to Human Activity and Viral DNA, Physica A, 265, 634-648, 1999.

Contoyiannis, Y. F. and Diakonos, F. K.: Criticality and intermittency in the order parameter space, Phys. Lett. A, 268, 286-292, 2000.

Dessler, A. J. and Parker, E. N.: Hydromagnetic theory of geomagnetic storms, J. Geophys. Res., 64, 2239-2252, 1959.

Dickman, R., Vespignani, A., and Zapperi, S.: Self-organized criticality as an absorbing-state phase transition, Phys. Rev. E, 57, 5095-5105, 1998.

Dobias, P.: Self-Organized Criticality in Asymmetric Warfare, Fractals, 17, 1-7, 2009.

Dobias, P., Voronkov, I. O., and Samson, J. C.: On the Nonlinear Plasma Instabilities During the Substorm Expansive Phase Onset, Phys. Plasmas, 11, 2046-2053, 2004.

Dobias, P., Wanliss, J. A., and Samson, J. C.: Nonlinear Stability of the Near-Earth Plasma sheet during substorms: February 9, 1995 event, Can. J. Phys., 84, 1029-1047, 2007.

Griffiths, R. B.: Rigorous Results and Theorems, in: Phase Transitions and Critical Phenomena, edited by: Domh, C. and Green, M. S., Academic Press, vol. 140, 1972.

Halley, J. D., Warden, A. C., Sadedin, S., and Li, W.: Rapid selforganized criticality: Fractal evolution in extreme environments,
Phys. Rev. E, 70, 036118, doi:10.1103/PhysRevE/70.036118, 2004.

Hurst, H. E.: Long-term storage capacity of reservoirs, Trans. Am. Soc. Civ. Eng., 116, 770-808, 1951.

Kantelhardt, J. W., Koscielny-Bunde, E., Rego, H. H. A., Havlin, S., and Bunde, A.: Detecting Long-range Correlations with Detrended Fluctuation Analysis, Physica A, 295, 441-454, 2001.

Lui, A. T. Y.: Multifractal and intermittent nature of substorm associated magnetic turbulence in the magnetotail, J. Atmos. Sol. Terr. Phys., 63, 1379-1385, 2001.

Lowen, S. B. and Teich, M. C.: Estimation and Simulation of Fractal Stochastic Point Processes, Fractals, 3, 183-210, 1995.

Murin, Yu., Ghetti, R., and Jakobsson, B.: Intermittency as a signal of criticality in multifragmentation studies, Phys. Rev. C, 55 , 528-531, 1997.

Samson, J. C., Rankin, R., and Tikhonchuk, V. T.: Optical signatures of auroral arcs produced by field line resonances: comparison with satellite observations and modeling, Ann. Geophys., 21, 933-945, 2003, http://www.ann-geophys.net/21/933/2003/.

Sckopke, N.: A general relation between the energy of trapped particles and the disturbance field near the Earth, J. Geophys. Res. 71, 3125-3130, 1966.

Shiokawa, K., Baumjohann, W., and Haerendel, G.: Braking of High-Speed Flows in the near-Earth Tail, Geophys. Res. Lett., 24, 1179-1182, 1997.

Sitnov, M. I., Sharma, A. S., Papadopoulos, K., and Vassiliadis, D.: Modeling substorm dynamics of the magnetosphere: From self-organization and self-organized criticality to nonequilibrium phase transitions, Phys. Rev. E 65, 016116, doi:10.1103/PhysRevE/65.016116, 2001.

Stepanova, M. V., Antonova, E. E., and Troshichev, O.: Intermittency of magnetospheric dynamics through non-Gaussian distribution function of PC-index fluctuations, Geophys. Res. Lett., 30, 1127, doi:10.1029/2002GL016070, 2003.

Stepanova, M. V., Antonova, E. E., Foppiano, A. J., Rosenberg, T. J., and Ovalle, E.: Intermittency of riometer auroral absorption observed at South Pole, J. Atmosph. Solar-Terrestrial Phys., 67(17-18), 1876-1884, doi:10.1016/j.jastp.2004.11.016, 2005.

Stepanova, M. V., Antonova, E. E., Foppiano, A. J., and Rosenberg, T. J.: Intermittency in the auroral absorption fluctuations as manifestation of magnetospheric turbulence, Adv. Space Res., 67, 1816-1884, doi:10.1016/jasr2005.04.112, 2005.

Thurner, S., Lowen, S. B., Feurstein, M. C., Heneghan, C., Feichtinger, H. C., and Teich, M. C.: Analysis, Synthesis, and Estimation of Fractal-Rate Stochastic Point Processes, Fractals, 5, 565-595, 1997.

Turcotte, D. L.: Fractals and Chaos in Geology and Geophysics, 2nd ed., Cambridge Univ. Press, Cambridge, UK, 1997.

Ukhorskiy, A. Y., Sitnov, M. I., Sharma, A. S., and Papadopoulos, K.: Global and multi-scale features of solar wind-magnetosphere coupling: From modeling to forecasting, Geophys. Res. Lett., 31, L08802, doi:10.1029/2003GL018932, 2004.

Uritsky, V. M. and Pudovkin, M. I.: Low frequency 1/f-like fluctuations of the AE-index as a possible manifestation of selforganized criticality in the magnetosphere, Ann. Geophys., 16, 1580-1588, 1998.

Wanliss, J. A., Anh, V. V., Yu, Z.-G., Watson, S., et al.: Multifractal modeling of magnetic storms via symbolic dynamics analysis. J. 
Geophys. Res., 110, A08214, doi:10.1029/2004JA010996, 2005. Wanliss, J. A. and Showalter, K. M.: High-resolution global storm index: $D_{s t}$ versus SYM-H, J. Geophys. Res., 111, A02202, doi:10.1029/2005JA011034, 2006.

Wanliss, J. A. and Dobias, P.: Space Storm as a Dynamic Phase Transition, J. Atmosph. Solar-Ter. Phys., 69, 675-684, 2007.

Wanliss, J. A. and Weygand, J. M.: Power law burst lifetime distribution of the SYM-H index, Geophys. Res. Lett., 34, L04107, doi:10.1029/2006GL028235, 2007.
Weygand, J. M., Kivelson, M. G., Khurana, K. K., Schwarzl, H. K., Thompson, S. M., McPherron, L., et al.: Plasma sheet turbulence observed by Cluster II, J. Geophys. Res., 110, A01205, doi:10.1029/2004JA010581, 2005.

Woodard, R., Newman, D. E., Sanchez, R., and Carreras, B. A.: Persistent dynamic correlations in self-organized critical systems away from their critical point, Physica A, 373, 215-230, 2006. 